\title{
Cetuximab in treatment of metastatic colorectal cancer: final survival analyses and extended RAS data from the NORDIC-VII study
}

Tormod Kyrre Guren ${ }^{1,2}$, Maria Thomsen ${ }^{1,3}$, Elin H Kure ${ }^{4}$, Halfdan Sorbye ${ }^{5,6}$, Bengt Glimelius ${ }^{7}$, Per Pfeiffer ${ }^{8,9}$, Pia Österlund ${ }^{10}$, Fridbjörn Sigurdsson ${ }^{11}$, Inger Marie Bowitz Lothe ${ }^{12}$, Astrid Marie Dalsgaard ${ }^{3}$, Eva Skovlund ${ }^{13}$, Thoralf Christoffersen ${ }^{14}$ and Kjell Magne Tveit ${ }^{*, 1,2,3}$

${ }^{1}$ Department of Oncology, Oslo University Hospital, Oslo, Norway; ${ }^{2}$ K.G. Jebsen Colorectal Cancer Research Centre, Oslo University Hospital, Oslo, Norway; ${ }^{3}$ Institute of Clinical Medicine, Faculty of Medicine, University of Oslo, Oslo, Norway; ${ }^{4}$ Department of Cancer Genetics, Institute for Cancer Research, Oslo University Hospital, Oslo, Norway; ${ }^{5}$ Department of Oncology, Haukeland University Hospital, Bergen, Norway; ${ }^{6}$ Department of Clinical Science, University of Bergen, Bergen, Norway; ${ }^{7}$ Department of Immunology, Genetics and Pathology, Uppsala University, Uppsala, Sweden; ${ }^{8}$ Department of Oncology, Odense University Hospital, Odense, Denmark; ${ }^{9}$ Institute of Clinical Research, University of Southern Denmark, Odense, Denmark; ${ }^{10}$ Department of Oncology, Tampere University Hospital, Tampere, Finland; ${ }^{11}$ Department of Oncology, Landspitali, Reykjavik, Iceland; ${ }^{12}$ Department of Pathology, Oslo University Hospital, Oslo, Norway; ${ }^{13}$ Department of Public Health and Nursing, Norwegian University of Science and Technology, Trondheim, Norway and ${ }^{14}$ Department of Pharmacology, Institute of Clinical Medicine, Faculty of Medicine, University of Oslo, Oslo, Norway

Background: The NORDIC-VII study is a randomised phase III trial of cetuximab plus continuous or intermittent fluorouracil, folinic acid, and oxaliplatin (Nordic FLOX) vs FLOX alone in first-line treatment of metastatic colorectal cancer. The present report presents an updated and final survival analysis with BRAF and extended RAS mutational status, 5 years after the primary analysis.

Methods: A total of 566 patients were included in the intention-to-treat (ITT) population of the NORDIC-VII study. Updated survival status was obtained from 176 patients who were alive in the primary survival analyses. Samples from 223 tumours previously found to be KRAS (exon 2) and BRAF (V600E) wild-type, were re-analysed for KRAS (exons 3 and 4) and NRAS (exons 2-4) mutations.

Results: Including the extended RAS analyses, RAS and BRAF mutational status was available from 457 patients (81\% of the ITT population). RAS was mutated in $46 \%$ and $B R A F$ in $12 \%$ of the tumours. RAS and BRAF, if mutated, were negative prognostic factors. The updated analyses confirmed the finding of the primary report that cetuximab did not provide any additional benefit when added to FLOX in patients with RAS/BRAF wild-type tumours, neither on progression-free nor overall survival. However, the outcomes in a subset of patients, which, after the first eight treatment cycles, received cetuximab alone, suggested a beneficial effect of cetuximab monotherapy.

Conclusions: Adding cetuximab to Nordic FLOX did not provide any clinical benefit, but the data suggested an effect of cetuximab monotherapy in patients with RAS/BRAF wild-type tumours in the NORDIC-VII cohort. The data were compatible with a negative interaction between cetuximab and the Nordic FLOX chemotherapy backbone.

*Correspondence: Professor KM Tveit; E-mail: kjetve@ous-hf.no 
Several studies have shown that adding EGFR antibodies to chemotherapy in first-line treatment of metastatic colorectal cancer (mCRC) improves outcome (Bokemeyer et al, 2009; Van Cutsem et al, 2009; Douillard et al, 2010). Initially, the effect was reported to be restricted to patients with KRAS (exon 2) wild-type tumours. Extended RAS analyses have demonstrated lack of response to EGFR antibodies also in patients with tumours harbouring other KRAS (exons 3 and 4) or NRAS mutations (Douillard et al, 2013; Bokemeyer et al, 2015; Van Cutsem et al, 2015).

The NORDIC-VII study (Tveit et al, 2012), randomised phase III trial, investigated the effect of adding cetuximab to a regimen of bolus 5-fluorouracil (5-FU)/folinic acid (FA) and oxaliplatin (Nordic FLOX) in first-line therapy of mCRC (Sorbye et al, 2004). In the intention-to-treat (ITT) population of 566 patients there was no statistically significant difference in overall response rate (ORR), progression-free survival (PFS), or overall survival (OS) between the treatment arms, and no effect of adding cetuximab to Nordic FLOX was demonstrated in patients with KRAS (exon 2) wild-type tumours (Tveit et al, 2012). These results were unexpected, however, the COIN trial also showed lack of effect when adding cetuximab to an oxaliplatin-capecitabine regimen in KRAS wild-type cases, while combination of cetuximab with the commonly used FOLFOX regimen gave a minor benefit (Maughan et al, 2011). Theoretically, the lack of effect of cetuximab in NORDIC-VII might have at least two explanations, a negative influence of the companion chemotherapy regimen used or a patient population in this study that was nonresponsive to anti-EGFR antibodies. On the basis of the results of a bolus 5-FU/FA regimen in the NORDIC-VII study, and the majority of patients in the COIN trial who received capecitabine, it has been suggested that when an EGFR antibody is added to fluoropyrimidine-oxaliplatin chemotherapy, the type of fluoropyrimidine regimen may influence treatment outcome (Grothey and Lenz, 2012; Mahipal and Grothey, 2016).

An updated OS analysis of NORDIC-VII is reported here, 5 years after the primary survival analysis. The impact of RAS (KRAS exons, 2, 3, and 4, and NRAS exons 2, 3, and 4) and BRAF (V600E) mutation status on prognosis and treatment outcome was investigated. Moreover, to investigate whether cetuximab might have an effect when given alone also in this patient population, we have examined the outcome in patients who received cetuximab in the absence of FLOX as maintenance therapy after eight cycles of treatment.

\section{MATERIALS AND METHODS}

Study design, treatments, and data collection. The NORDIC-VII study is a randomised phase III trial investigating the effect of adding cetuximab to the Nordic FLOX regimen of bolus 5-FU/FA and oxaliplatin in previously untreated patients with mCRC. Patients were randomly assigned to receive FLOX (arm A), cetuximab plus FLOX (arm B), or continuous cetuximab combined with intermittent FLOX (arm C). In arms A and B, treatment was continued until progressive disease (PD) or unacceptable toxicity. In arm C, FLOX was usually stopped after eight courses (16 weeks of treatment), and in cases of objective response or stable disease, cetuximab was continued as maintenance therapy. After recording $\mathrm{PD}$ in arm C, FLOX was reintroduced and continued together with cetuximab until PD or unacceptable toxicity (Tveit et al, 2012). Between May 2005 and October 2007, 571 patients from 32 centres were enrolled in the study and 566 were evaluable in the ITT population. In the primary report, patients were followed for PFS and OS analyses up to 12 and 18 months, respectively, after inclusion of the last patient (Tveit et al, 2012).

Updated OS data were obtained from 176 out of 182 patients reported alive in the primary analysis. The updated OS analysis had censoring date 30 April 2014. Four patients (three in arm A and one in arm C) were lost to follow-up and two had withdrawn their consent before the primary analysis, and for these the

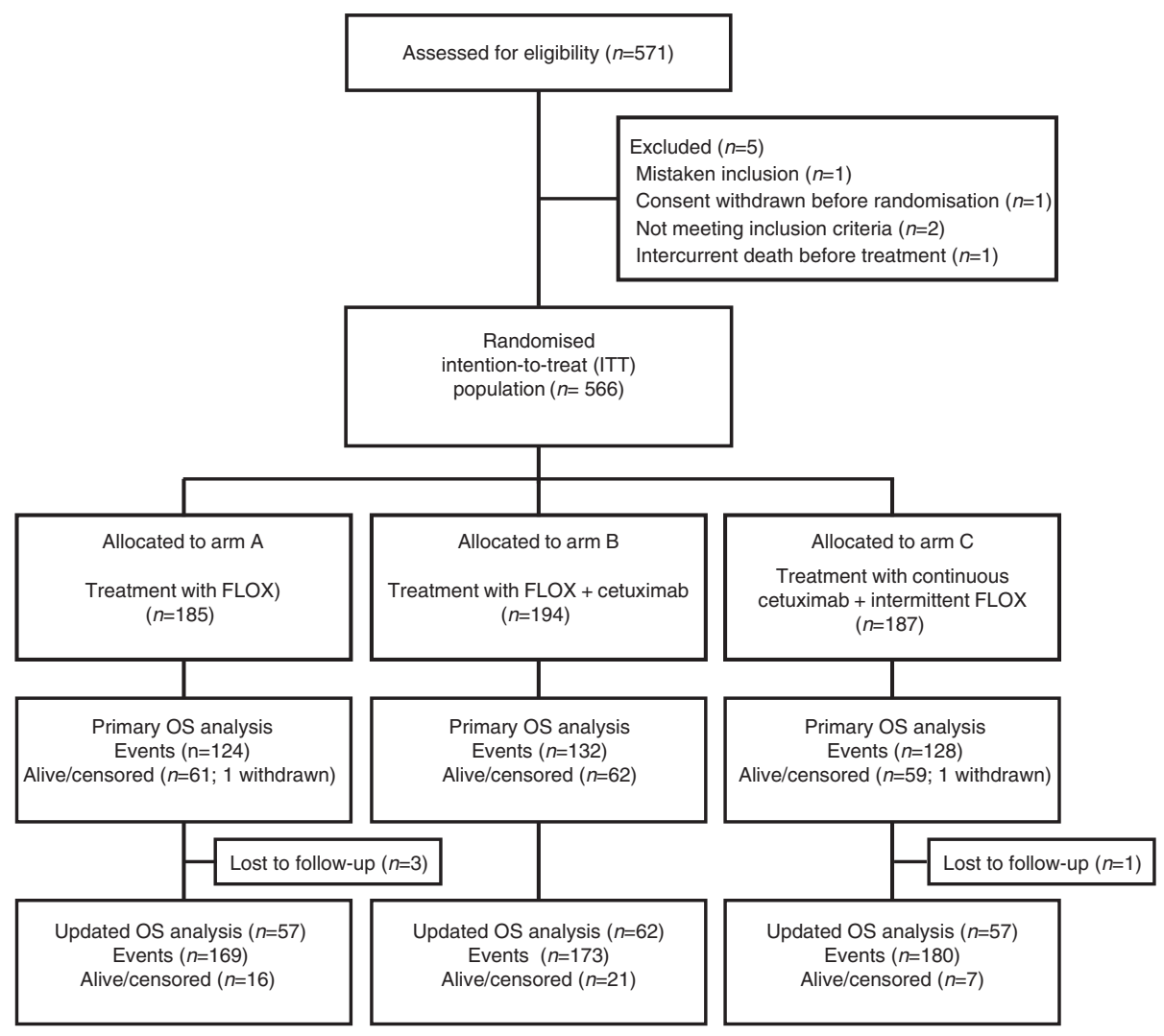

Figure 1. CONSORT diagram of the study design. 
censoring date was the last date they were known to be alive and not withdrawn (Figure 1).

Mutation analysis of KRAS, NRAS, and BRAF. In the primary analysis, KRAS (exon 2) mutation status was available for 498 patients. From this cohort with known KRAS (exon 2, codons G12D, G12A, G12V, G12S, G12C, G12R, and codon G13D) and $B R A F$ (exon 15, V600E) mutation status, tumour DNA of 457 patients ( $81 \%$ of the ITT population) was available for extended RAS mutation testing. In all, 179 patients $(39 \%)$ had tumours with known KRAS (exon 2) mutations, and 55 (12\%) had tumours with $B R A F$ mutation. As KRAS and BRAF mutations were considered to be mutually exclusive (Rajagopalan et al, 2002), tumour DNA from the remaining $223 K R A S$ (exon 2)/BRAF wild-type patients was analysed for the other KRAS mutations (in exons 3 and 4) and NRAS mutations (in exons 2, 3, and 4).

Tumour DNA extraction from formalin-fixed, paraffinembedded (FFPE) tumour tissue and analyses of mutations in KRAS (exon 2) and BRAF have been previously reported (Tveit et al, 2012). DNA was screened for the presence of the KRAS mutations Q61H, Q61L, and Q61R in exon 3, and K117X (K117N $351 \mathrm{~A}>\mathrm{C}, \mathrm{K} 117 \mathrm{~N} 351 \mathrm{~A}>\mathrm{T}$, and K117R, K117E) and A146X
(A146T, A146P, and A146V) in exon 4, using the KRAS Mutation Analysis Kit for Real-Time PCR (exons 2, 3, and 4) by EntroGen (Woodland Hills, CA, USA). The NRAS mutations G12C, G12D, G12S, G13V, G13R, Q61K, Q61R, Q61L, Q61H, and A146T in exons 2, 3, and 4 were analysed using the NRAS Mutation Analysis Kit (EntroGen). The mutation detection assays and the analysis of the results were done in accordance with the manufacturer's instructions. Input in the KRAS (exons 3 and 4) and NRAS assays were 10 and $20 \mathrm{ng}$ of whole-genome DNA from FFPE material, respectively.

Ethics. The NORDIC-VII study (http://clinicaltrials.gov/show/ NCT00145314) was approved by the national ethics committees and governmental authorities in each country and conducted in accordance with the Declaration of Helsinki. All patients had provided written informed consent.

Statistical analysis. The statistical analyses, using IBM SPSS (version 23, IBM Corp., Armonk, NY, USA), were performed in the ITT population and in subsets of patients based on RAS and $B R A F$ mutation status. Demographic data were described with median and range (continuous variables) and proportions and

Table 1. Baseline patient demographics and clinical characteristics

\begin{tabular}{|c|c|c|c|c|c|c|c|c|c|c|}
\hline & \multicolumn{10}{|c|}{ Study population } \\
\hline & \multirow{2}{*}{$\begin{array}{c}\text { ITT } \\
\text { Total } \\
(n=566)\end{array}$} & \multirow{2}{*}{$\begin{array}{c}\text { RAS/ } \\
\text { BRAF } \\
\text { status } \\
\text { unknown } \\
\text { Total } \\
(n=109) \\
\end{array}$} & \multicolumn{4}{|c|}{ Tested for RAS and BRAF mutations } & \multicolumn{4}{|c|}{ RAS/BRAF wild-type } \\
\hline & & & $\begin{array}{c}\text { Total } \\
(n=457)\end{array}$ & $\begin{array}{c}\text { Arm A } \\
(n=146)\end{array}$ & $\begin{array}{c}\text { Arm B } \\
(n=158)\end{array}$ & $\begin{array}{c}\text { Arm C } \\
(n=153)\end{array}$ & $\begin{array}{c}\text { Total } \\
(n=192)\end{array}$ & $\begin{array}{c}\text { Arm A } \\
(n=64)\end{array}$ & $\begin{array}{c}\text { Arm B } \\
(n=66)\end{array}$ & $\begin{array}{l}\text { Arm C } \\
(n=62)\end{array}$ \\
\hline \multicolumn{11}{|l|}{ Age (years) } \\
\hline Median (range) & $62(24-75)$ & $62(30-75)$ & $62(24-75)$ & $60(35-75)$ & $61(24-74)$ & $64(33-75)$ & $62(24-75)$ & $60(40-75)$ & $60(24-74)$ & $65(36-75)$ \\
\hline \multicolumn{11}{|l|}{ Gender, n (\%) } \\
\hline Male & $334(59)$ & $61(56)$ & $273(60)$ & $80(55)$ & $98(62)$ & $95(62)$ & $126(66)$ & $30(47)$ & $51(77)$ & $45(73)$ \\
\hline Female & $232(41)$ & $48(44)$ & $184(40)$ & $66(45)$ & $60(38)$ & $58(38)$ & $66(34)$ & $34(53)$ & $15(23)$ & $17(27)$ \\
\hline \multicolumn{11}{|l|}{ WHO PS, $n(\%)$} \\
\hline 0 & $380(67)$ & $73(67)$ & $307(67)$ & $102(70)$ & $108(68)$ & $97(63)$ & $129(67)$ & $48(75)$ & $44(67)$ & $37(60)$ \\
\hline 1 & $162(29)$ & $32(29)$ & $130(28)$ & $38(26)$ & $44(28)$ & $48(31)$ & $54(28)$ & $11(17)$ & $19(29)$ & $24(39)$ \\
\hline 2 & $24(4)$ & $4(4)$ & $20(4)$ & $6(4)$ & $6(4)$ & $8(5)$ & $9(5)$ & $5(8)$ & $3(5)$ & $1(2)$ \\
\hline \multicolumn{11}{|c|}{ Site of primary tumour, $n(\%)$} \\
\hline Colon & $333(59)$ & $58(53)$ & $275(60)$ & $83(57)$ & $91(58)$ & $101(66)$ & $111(58)$ & $36(56)$ & $33(50)$ & $42(68)$ \\
\hline Rectum & $233(41)$ & $51(47)$ & $182(40)$ & $63(43)$ & $67(42)$ & $52(34)$ & $81(42)$ & $28(44)$ & $33(50)$ & $20(32)$ \\
\hline \multicolumn{11}{|c|}{ Previous treatment, $n(\%)$} \\
\hline Primary tumour resected & $382(68)$ & $35(32)$ & $347(76)$ & $114(78)$ & $118(75)$ & $115(75)$ & $146(76)$ & $51(80)$ & $49(74)$ & $46(74)$ \\
\hline Adjuvant chemotherapy & $51(9)$ & $5(5)$ & $46(10)$ & $14(10)$ & $16(10)$ & $16(11)$ & $21(11)$ & $6(9)$ & 9 (14) & $6(10)$ \\
\hline Preoperative radiotherapy & $80(14)$ & $10(9)$ & $70(15)$ & $18(12)$ & $29(18)$ & $23(15)$ & $29(15)$ & $6(9)$ & $17(26)$ & $6(10)$ \\
\hline \multicolumn{11}{|c|}{ Timing of metastases, $n(\%)$} \\
\hline Synchronous & $402(71)$ & $94(86)$ & $308(67)$ & $97(66)$ & $106(67)$ & $105(69)$ & $129(67)$ & $43(67)$ & $45(68)$ & $41(66)$ \\
\hline Metachronous & $164(29)$ & $15(14)$ & 149 (33) & $49(34)$ & $52(33)$ & $48(31)$ & $63(33)$ & $21(33)$ & $21(32)$ & $21(34)$ \\
\hline \multicolumn{11}{|c|}{ Number of metastatic sites, $n(\%)$} \\
\hline 1 site & $162(29)$ & $26(24)$ & $136(30)$ & $44(30)$ & $53(34)$ & $39(26)$ & $67(35)$ & $24(38)$ & $23(35)$ & $20(32)$ \\
\hline$>1$ sites & $404(71)$ & $83(76)$ & $321(70)$ & $102(70)$ & $105(67)$ & $114(75)$ & $125(65)$ & $40(63)$ & $43(65)$ & $42(68)$ \\
\hline \multicolumn{11}{|c|}{ Type of metastases, $n$ (\%) } \\
\hline Liver only & 107 (19) & $17(16)$ & $90(20)$ & $31(21)$ & $34(22)$ & $25(16)$ & $53(28)$ & $19(29)$ & $17(26)$ & $17(27)$ \\
\hline Liver plus other & $312(55)$ & $69(63)$ & $243(53)$ & $69(47)$ & $76(48)$ & $98(64)$ & $92(48)$ & 25 (39) & $31(47)$ & $36(58)$ \\
\hline Non-liver & $147(26)$ & $23(21)$ & $124(27)$ & $46(32)$ & $48(30)$ & $30(20)$ & $47(25)$ & $20(31)$ & $18(27)$ & $9(15)$ \\
\hline \multicolumn{11}{|l|}{ Investigations, n (\%) } \\
\hline $\begin{array}{l}\text { Normal alkaline } \\
\text { phosphatase level }\end{array}$ & $298(53)$ & $48(44)$ & $250(55)$ & $86(59)$ & $82(52)$ & $82(54)$ & $100(52)$ & $38(59)$ & $34(52)$ & $28(45)$ \\
\hline Platelet count $<400 \mathrm{nl}^{-1}$ & $398(70)$ & $70(64)$ & $328(72)$ & $100(69)$ & $115(73)$ & $113(74)$ & $142(74)$ & $45(70)$ & $51(77)$ & $46(74)$ \\
\hline $\begin{array}{l}\text { White blood cell count } \\
<10 \mathrm{nl}^{-1}\end{array}$ & $428(76)$ & $69(63)$ & $359(79)$ & $118(81)$ & $123(78)$ & $118(77)$ & $152(79)$ & $51(80)$ & $53(80)$ & $48(77)$ \\
\hline
\end{tabular}


percentages (categorical variables). The PFS and OS were compared between treatment groups using the log-rank test, and treatment effects were estimated using Kaplan-Meier plots and Cox proportional hazards model. Separate univariable analyses of the effect of the WHO performance status, alkaline phosphatase (ALP), and RAS and BRAF mutation status were performed. Only variables statistically significant in univariable analyses were included in the multivariable analyses, and models were thereafter restricted to include statistically significant variables only.

\section{RESULTS}

Patients. In all, 566 patients were evaluable and included in the ITT population in the primary analysis of the NORDIC-VII study. Baseline characteristics were well balanced between the three treatment arms (Tveit et al, 2012). In the present study of 457 patients with available results for RAS and BRAF mutation status, the patient groups were well balanced and representative for the ITT population (Table 1 and Supplementary Table S1).

Updated OS. The median OS for the overall ITT population $(n=566)$ was 20.1 months, and there was no statistically

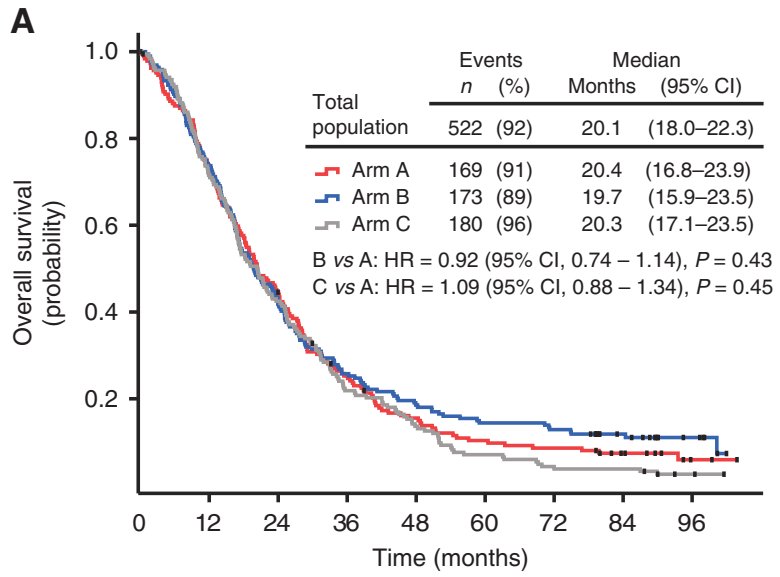

\begin{tabular}{lllllllrrr}
\multicolumn{2}{l}{ No. at risk } \\
Arm A & 185 & 135 & 81 & 45 & 27 & 18 & 15 & 9 & 2 \\
Arm B & 194 & 143 & 81 & 50 & 36 & 28 & 25 & 15 & 6 \\
Arm C & 187 & 133 & 79 & 40 & 25 & 13 & 8 & 7 & 2
\end{tabular}

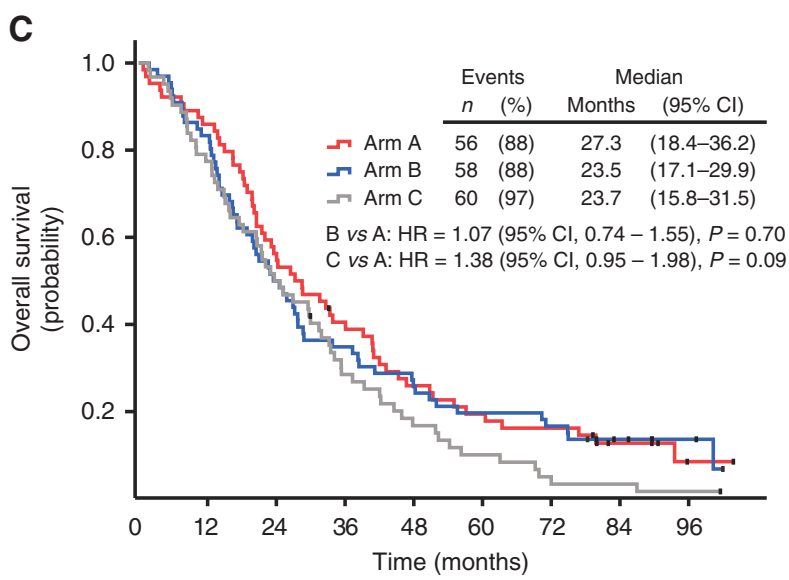

No. at risk

$\begin{array}{llllllllll}\text { Arm A } & 64 & 55 & 36 & 25 & 16 & 12 & 10 & 5 & 1 \\ \text { Arm B } & 66 & 55 & 33 & 23 & 17 & 13 & 11 & 6 & 3\end{array}$

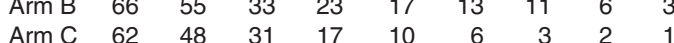

significant difference between the treatment arms (Figure 2A). In all, 38 out of 44 censored patients were registered alive in the updated analysis, 12 (7\%), 21 (11\%), and $5(3 \%)$ in arm A, B, and $\mathrm{C}$, respectively. Of the 38 patients reported alive, 18 (47\%) had stopped study treatment due to secondary complete surgical resection of metastases, 5 of 12 in arm A, 11 of 21 in $\operatorname{arm~B,~and~} 2$ of 5 in arm C.

Extended RAS and BRAF mutations. The mutation analyses are reported in Table 2. In the cohort of 457 patients, 210 (46\%) had tumours with (any) RAS mutations, 55 (12\%) had BRAF mutation, and 192 (42\%) were RAS/BRAF wild-type. The frequency and distribution of $R A S$ and $B R A F$ mutations were similar in the three treatment arms A, B, and C, with any RAS mutations in $63(43 \%)$, $72(46 \%)$, and $75(49 \%)$, respectively, and BRAF mutations in 19 (13\%), 20 (13\%), and $16(10 \%)$, respectively.

Survival related to $R A S$ and $B R A F$ mutation status. The patient population with known $R A S$ and $B R A F$ mutation status had almost identical OS compared to the ITT population (Figure 2B), and, as shown in Figure 2C, there was no statistically significant difference between the treatment arms in patients with $R A S / B R A F$ wild-type tumours $(27.3,23.5$, and 23.7 months, respectively). These data are

B

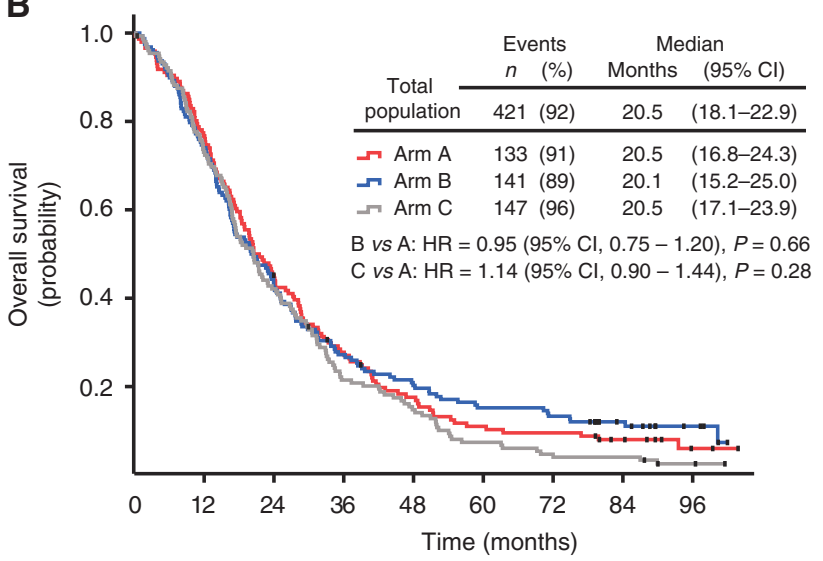

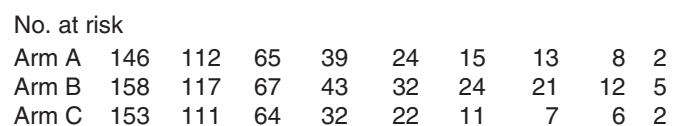

D

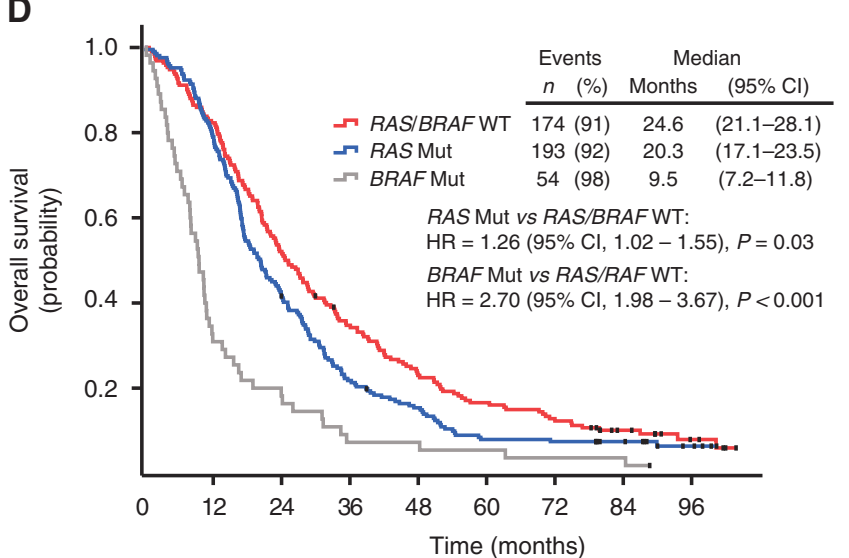

No. at risk $\begin{array}{llllllllll}\text { RAS/BRAF WT } & 192 & 158 & 100 & 65 & 43 & 31 & 24 & 13 & 5\end{array}$ $\begin{array}{llllllllll}\text { RAS Mut } & 210 & 165 & 86 & 45 & 31 & 16 & 15 & 11 & 4\end{array}$ $\begin{array}{llllllllll}\text { BRAF Mut } & 55 & 17 & 10 & 4 & 4 & 3 & 2 & 2 & -\end{array}$

Figure 2. Survival curves. Overall survival in the three different treatment arms: (A) intention-to-treat population; (B) RAS- and BRAF-tested population; and (C) RAS and BRAF wild-type population. (D) Overall survival in patients with RAS and BRAF wild-type, RAS mutant, and BRAFmutated tumours. $\mathrm{Cl}=$ confidence interval; $\mathrm{HR}=$ hazard ratio; Mut = mutation; $\mathrm{WT}=$ wild-type. 
in agreement with the previously published findings (Tveit et al, 2012).

Prognostic value of RAS and BRAF mutations. The outcome in the patients, depending on the mutational status of their tumours, is shown in Figure 2D. The median OS for those with $R A S / B R A F$ wild-type tumours was 24.6 months, while it was shorter for those with tumours harbouring mutations in RAS (20.3 months) and, particularly, in patients with $B R A F$-mutated tumours (9.5 months). The prognostic value of $R A S$ or $B R A F$ mutation was further demonstrated in a Cox regression model for OS, which included the WHO performance status and ALP level registered before start of treatment as explanatory variables. Similar results were obtained when OS was censored at start of treatment with EGFR antibodies as second- or third-line therapy (Supplementary Table S2).

Response rates and PFS related to RAS and BRAF mutation status. Confirmed ORR values in the ITT population, the RAS- and $B R A F$-tested population, and subgroups by mutation status are listed in Supplementary Table S3. Numerically, ORR was highest in patients with $R A S / B R A F$ wild-type tumours, and in this cohort ORR was somewhat higher (not statistically significant) for patients in arm B (61\%) or arm C (60\%), compared to those in arm A (50\%).

The median PFS was 8.3, 8.2, and 7.4 months for arms A, B, and $\mathrm{C}$, respectively, in the population with known $R A S$ and $B R A F$ mutation status (Figure $3 \mathrm{~A}$ ). In patients with $R A S / B R A F$ wild-type tumours, the curves for arms $\mathrm{A}, \mathrm{B}$, and $\mathrm{C}$ were similar, with median PFS of 9.3, 9.5, and 9.2 months, respectively (Figure 3B). In patients with RAS mutant tumours, there was no statistically significant difference between the median PFS of 8.3 months in arm B compared to 7.9 months in arm A (HR, $0.84(0.59-1.19) ; P=0.32)$.

Progression-free survival in patients treated with cetuximab in the absence of FLOX. From the start of treatment, patients in arm A were randomised to receive FLOX, while those in both arms B and $C$ received cetuximab plus FLOX. After eight cycles of chemotherapy $(\sim 16$ weeks of treatment), the patients in arm $C$ were treated with cetuximab only, while those in arm A continued the therapy with FLOX. This fact allowed a comparison of the outcome of cetuximab monotherapy with FLOX treatment from cycle 9 of 159 patients with known RAS and BRAF mutation status. Progression-free survival was calculated from the start of cycle 9 to event (progression or death) or censoring. Figure 3C shows that for patients with $R A S / B R A F$ wild-type tumours the outcome in arm A and $C$ was closely similar, 5.8 vs 6.3 months. In contrast, for patients with RAS-mutated tumours, PFS was markedly shorter in those receiving cetuximab monotherapy (arm C) compared to patients treated with FLOX ( $\operatorname{arm~A),~} 2.3$ vs 4.4 months $(P=0.003$, Figure $3 \mathrm{D})$. There was a positive interaction between treatment arms, $\mathrm{A}$ and $\mathrm{C}$, and $R A S / B R A F$ mutation status $(P=0.046)$

Third-line treatment with EGFR antibodies. Figure 4 shows the treatment outcome of 82 patients with known RAS and BRAF mutation status who received therapy with EGFR antibodies (cetuximab or panitumumab) as second- or third-line therapy after end of treatment in NORDIC-VII. The patients with $R A S / B R A F$ wildtype tumours had a median survival of 10.1 months from start of later-line treatment with EGFR antibodies, while the median survival of patients with RAS or BRAF mutant tumours was 6.6 months.

Colon vs rectum. Outcome stratified on the origin of primary tumour was examined (Supplementary Table S4). In the ITT population, patients with colon cancer had shorter median OS compared to those with their primary tumour in rectum, 18.6 vs 22.3 months (HR, $0.83(0.70-0.99) ; P=0.04)$. A similar trend was observed in subpopulations based on RAS/BRAF mutation status. There was no statistically significant difference in PFS or OS between the treatment arms neither in colon nor in rectal cancer. Location of primary tumour within colon was not registered in this study.
Table 2. RAS and BRAF mutation status

\begin{tabular}{|c|c|c|c|c|}
\hline \multirow[b]{2}{*}{ Genotype } & \multicolumn{4}{|c|}{ Study population } \\
\hline & $\begin{array}{c}\text { Total } \\
(n=457) \\
n(\%)\end{array}$ & $\begin{array}{c}\text { Arm A } \\
(n=146) \\
n(\%)\end{array}$ & $\begin{array}{c}\text { Arm B } \\
(n=158) \\
n(\%)\end{array}$ & $\begin{array}{c}\text { Arm C } \\
(n=153) \\
n(\%)\end{array}$ \\
\hline $\begin{array}{l}\text { Wild-type (RAS and } \\
\text { BRAF) }\end{array}$ & $192(42.0)$ & $64(43.8)$ & $66(41.8)$ & $62(40.5)$ \\
\hline RAS mutant & $210(46.0)$ & $63(43.2)$ & $72(45.6)$ & $75(49.0)$ \\
\hline KRAS exon 2 mutant & $179(39.2)$ & $55(37.7)$ & $67(42.4)$ & $57(37.3)$ \\
\hline KRAS G12A & $16(3.5)$ & $5(3.4)$ & $5(3.2)$ & $6(3.9)$ \\
\hline KRAS G12R & $3(0.7)$ & $1(0.7)$ & $1(0.6)$ & $1(0.7)$ \\
\hline KRAS G12D & $62(13.6)$ & $14(9.6)$ & $27(17.1)$ & 21 (13.7) \\
\hline KRAS G12C & $16(3.5)$ & $4(2.7)$ & $7(4.4)$ & $5(3.3)$ \\
\hline KRAS G12S & $11(2.4)$ & $5(3.4)$ & $4(2.5)$ & $2(1.3)$ \\
\hline KRAS G12V & $30(6.6)$ & $11(7.5)$ & $11(7.0)$ & $8(5.2)$ \\
\hline KRAS G13D & $41(9.0)$ & $15(10.3)$ & $12(7.6)$ & $14(9.2)$ \\
\hline KRAS exon 3 mutant & $9(2.0)$ & $2(1.4)$ & $2(1.3)$ & $5(3.3)$ \\
\hline KRAS Q61H & $3(0.7)$ & 0 & $1(0.6)$ & $2(1.3)$ \\
\hline KRAS Q61L & $5(1.1)$ & $2(1.4)$ & $1(0.6)$ & $2(1.3)$ \\
\hline KRAS Q61R & $1(0.2)$ & 0 & 0 & $1(0.7)$ \\
\hline KRAS exon 4 mutant & $7(1.5)$ & $2(1.4)$ & $1(0.6)$ & $4(2.6)$ \\
\hline KRAS K117X & $1(0.2)$ & 0 & 0 & $1(0.7)$ \\
\hline KRAS A146X & $6(1.3)$ & $2(1.4)$ & $1(0.6)$ & $3(2.0)$ \\
\hline NRAS exon 2 mutant & $8(1.8)$ & $2(1.4)$ & $1(0.6)$ & $5(3.3)$ \\
\hline NRAS G12S & $1(0.2)$ & $1(0.7)$ & 0 & 0 \\
\hline NRAS G12C & $6(1.3)$ & 0 & $1(0.6)$ & $5(3.3)$ \\
\hline NRAS G13V & $1(0.2)$ & $1(0.7)$ & 0 & 0 \\
\hline NRAS exon 3 mutant & $7(1.5)$ & $2(1.4)$ & $1(0.6)$ & $4(2.6)$ \\
\hline NRAS Q61L & $2(0.4)$ & 0 & 0 & $2(1.3)$ \\
\hline NRAS Q61K & $3(0.7)$ & $1(0.7)$ & $1(0.6)$ & $1(0.7)$ \\
\hline NRAS Q61R & $2(0.4)$ & $1(0.7)$ & 0 & $1(0.7)$ \\
\hline BRAF mutant (V600E) & 55 (12.0) & $19(13.0)$ & $20(12.7)$ & $16(10.5)$ \\
\hline
\end{tabular}

\section{DISCUSSION}

The updated survival analysis showed no statistically significant difference between OS for the three treatment arms, in the ITT, $R A S / B R A F$ tested, or $R A S / B R A F$ double wild-type populations. Similarly, in the population of patients with known $R A S$ and $B R A F$ mutation status and in patients with $R A S / B R A F$ double wild-type tumours, there was no statistically significant difference in PFS between the treatment arms. However, in arm B there was a numerically higher number of long-term survivors and a higher number of patients with complete surgical resection of metastases. In the primary analysis of NORDIC-VII an unexpected trend towards increased PFS was found in patients with tumours harbouring KRAS (exon 2) mutations who received FLOX plus cetuximab, as compared to FLOX alone (arm B vs arm A). This difference in PFS was less pronounced in analyses based on the extended RAS mutation status, further suggesting that the previously reported result was an incidental finding. Taken together, the analyses based on RAS status and updated survival data did not change the conclusion from the primary report that cetuximab did not add any significant benefit to the Nordic FLOX regimen in first-line treatment of mCRC (Tveit et al, 2012).

Patients with colon cancer had shorter median OS compared to those with rectal cancer, in line with other trials (Tejpar et al, 2017). Recently, pooled data from two randomised phase III trials have emphasised that clinical benefit of cetuximab treatment is significantly higher in patients with left-sided colon and rectum tumours compared to right-sided (Tejpar et al, 2017). Precise location of the primary tumour within colon was not registered in this study, and as no effect of adding cetuximab to FLOX was observed in the population of colon or rectal cancer patients in NORDIC-VII, a possible difference in outcome between right and left colon cancer was not further examined. 
A

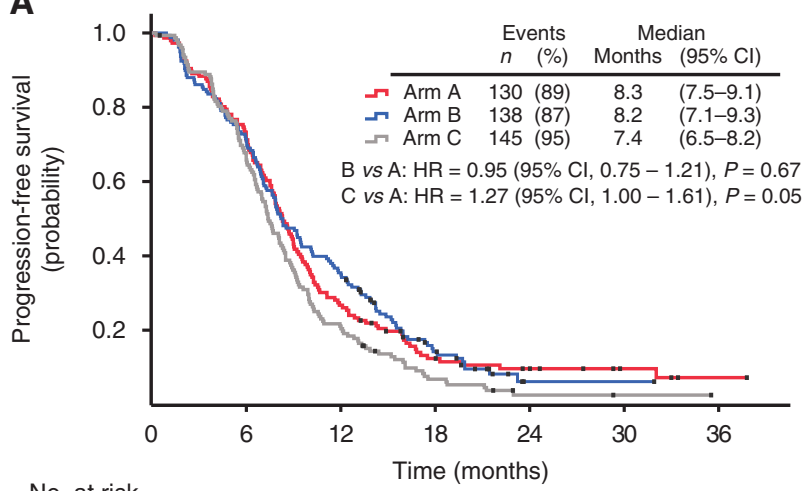

No. at risk

$\begin{array}{lrrrrrrr}\text { Arm A } & 146 & 104 & 39 & 15 & 9 & 4 & 1 \\ \text { Arm B } & 158 & 112 & 56 & 17 & 1 & 1 & - \\ \text { Arm C } & 153 & 100 & 32 & 9 & 2 & 1 & -\end{array}$

C

\section{8 cycles \\ (16 weeks)}

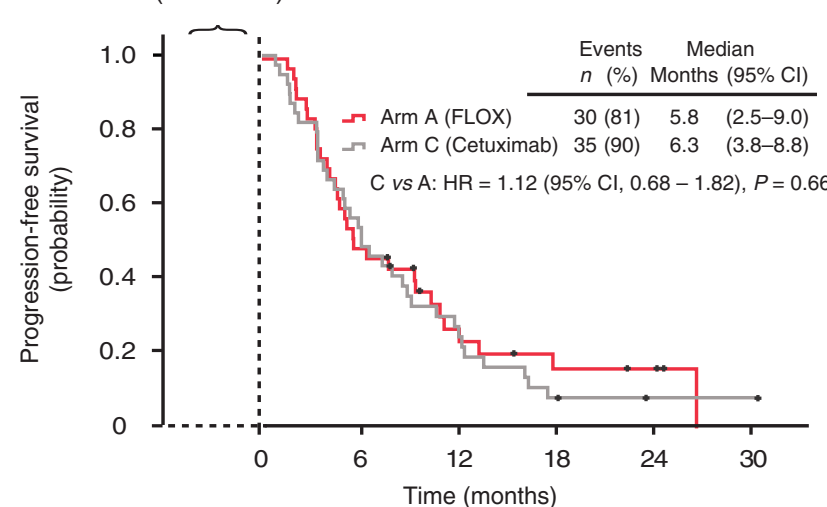

No. at risk

$\begin{array}{lrrrrrr}\text { Arm A } & 37 & 18 & 8 & 5 & 3 & - \\ \text { Arm C } & 39 & 22 & 11 & 4 & 2 & 1\end{array}$
B

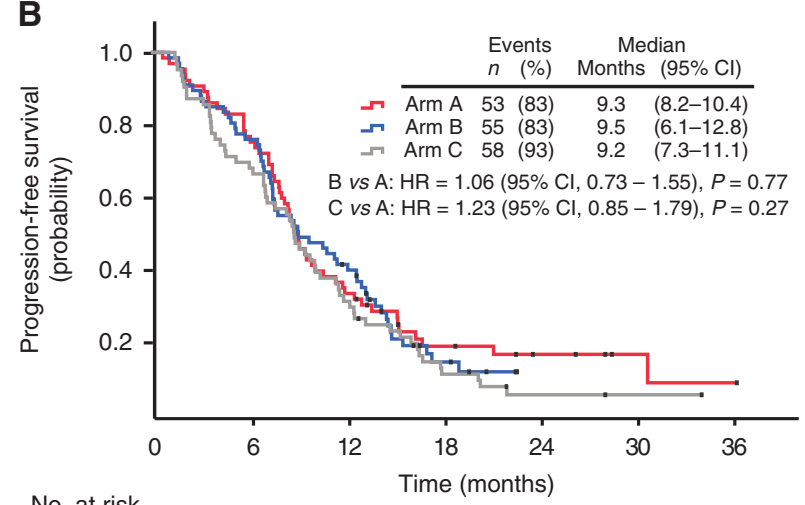

No. at risk

$\begin{array}{llllllll}\text { Arm A } & 64 & 50 & 23 & 9 & 6 & 2 & 1 \\ \text { Arm B } & 66 & 51 & 28 & 7 & - & - & - \\ \text { Arm C } & 62 & 43 & 22 & 8 & 2 & 1 & -\end{array}$

\section{D}

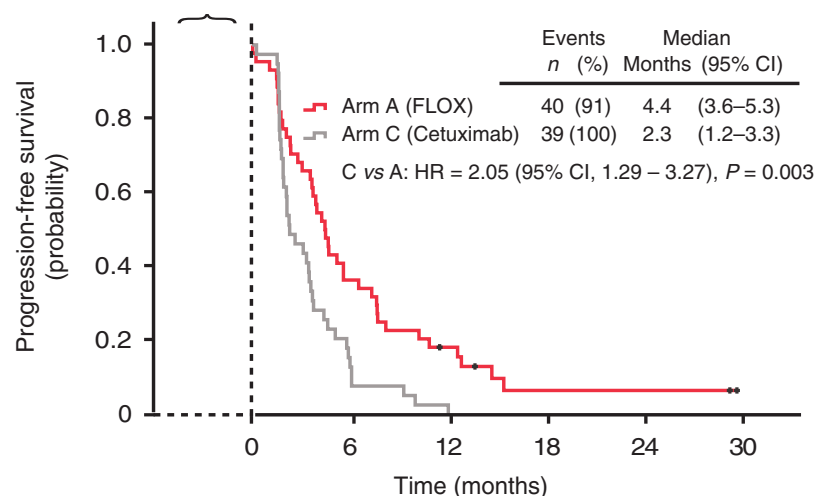

$\begin{array}{lrrrrrr}\text { No. at risk } & & & & & & \\ \text { Arm A } & 44 & 16 & 7 & 2 & 2 & - \\ \text { Arm C } & 39 & 5 & - & -\end{array}$

Figure 3. Survival curves. Progression-free survival in the three different treatment arms: (A) RAS- and BRAF-tested population; (B) RAS and BRAF wildtype population. Progression-free survival from start of treatment cycle 9 in arms A (FLOX) and C (maintenance treatment with cetuximab) in patients with (C) RAS/BRAF wild-type tumours and (D) RAS-mutated tumours. $\mathrm{Cl}=$ confidence interval; $\mathrm{HR}=$ hazard ratio.

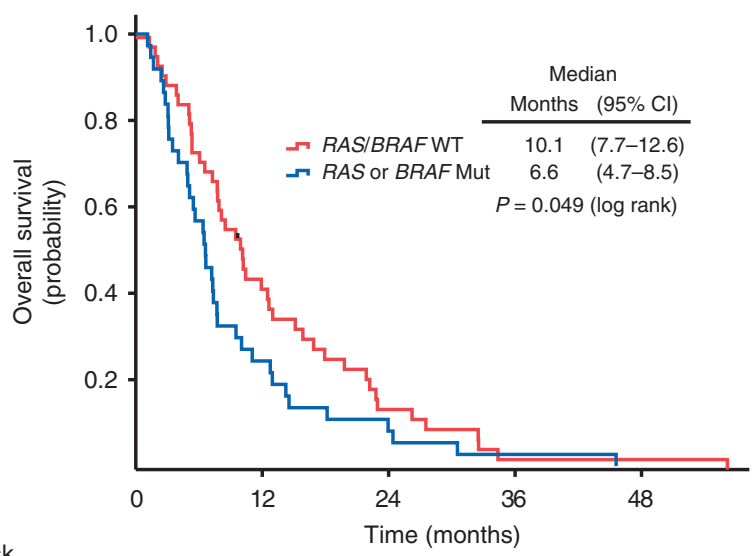

No. at risk

$\begin{array}{llclll}R A S / B R A F W T & 45 & 18 & 6 & 1 & 1 \\ \text { RAS or BRAF Mut } & 37 & 9 & 3 & 1 & -\end{array}$

Figure 4. Kaplan-Meier estimate of overall survival from start of second- or third-line treatment with EGFR antibodies, cetuximab $(n=75)$, or panitumumab $(n=7)$, in 82 patients with known RAS/BRAF status. Mut = mutation; $\mathrm{WT}=$ wild-type.

The frequency of the RAS mutations was in agreement with those reported in other trials (Douillard et al, 2013; Bokemeyer et al, 2015; Van Cutsem et al, 2015), and patients with tumours harbouring RAS or BRAF mutations showed impaired outcomes compared to those with $R A S / B R A F$ wild-type tumours, also in line with results reported from recent first-line chemotherapy trials in mCRC (Maughan et al, 2011; Cremolini et al, 2015). Thus, it is unlikely that the reason for lack of effect of cetuximab in this trial was that the NORDIC-VII cohort differed fundamentally from other patient populations studied.

After eight cycles of chemotherapy, the patients in arm $\mathrm{C}$ were treated with cetuximab only, while those in arm A continued the therapy with FLOX. This study design allowed a comparison of cetuximab monotherapy with FLOX treatment from cycle 9. In patients with double wild-type tumours the PFS from cycle 9 was quite similar for the patients receiving FLOX (arm A) and those treated with cetuximab only ( $\operatorname{arm} \mathrm{C}$ ). In contrast, in patients with $R A S$-mutated tumours, the PFS was significantly reduced in those who received cetuximab monotherapy compared with FLOX. On the basis of the assumption that chemotherapy (FLOX) received from cycle 9 improved the outcome in patients with $R A S / B R A F$ wild-type as well as in RAS-mutated tumours, these results strongly suggest that also for the NORDIC-VII cohort, in patients with $R A S / B R A F$ wild-type tumours, unlike $R A S$-mutated, cetuximab may exert an effect as single agent. These results also suggest that maintenance cetuximab therapy, as given in arm $\mathrm{C}$, provides an alternative approach to ongoing chemotherapy in the relevant biomarker-selected group, in line with the results of the COIN-B trial (Wasan et al, 2014). 
To further elucidate the effects of anti-EGFR therapy in this patient population, survival of patients who received such therapy as part of second- or third-line treatment was analysed. This treatment was mainly offered before the demonstration that effect of EGFR antibodies was restricted to KRAS wild-type tumours (Amado et al, 2008; Karapetis et al, 2008). Patients with $R A S / B R A F$ wild-type tumours, previously treated in NORDICVII, had significantly longer median OS from start of this treatment compared to those with RAS or BRAF mutations, consistent with outcome reported by others (Amado et al, 2008; Karapetis et al, 2008). These results are based on observational data and should be interpreted with caution as RAS and BRAF mutations affect prognosis and comparison with no EGFR antibody therapy was not possible. However, it may be hypothesised that a 3.5 month difference in median OS from start of second- or third-line EGFR antibody therapy reflects some effect of this treatment in the wild-type patients. The prognosis reflected by the RAS/BRAF mutation status was sustained in a Cox regression model where OS was censored at start of second- or third-line treatment with EGFR antibodies if the patients had received such treatment.

In view of the results of NORDIC-VII, together with the data from COIN and the New EPOC trials (Maughan et al, 2011; Primrose et al, 2014), as compared to trials where cetuximab was combined with irinotecan-based regimens (Van Cutsem et al, 2009), it may be discussed whether cetuximab and oxaliplatin are good partners. We have no clinical data to answer this question directly. However, preclinical studies have mechanistically demonstrated an antagonistic modulation of cetuximab on the effect of oxaliplatin (Dahan et al, 2009; Santoro et al, 2015). It should, however, be noted that in the TAILOR trial a significant benefit in terms of ORR, PFS, and OS was reported with the addition of cetuximab to FOLFOX (Qin et al, 2016). It has been suggested that the type of fluoropyrimidine regimen has influence on treatment outcome when an EGFR antibody is added to fluoropyrimidine-oxaliplatin chemotherapy (Grothey and Lenz, 2012), and so far, a benefit has only been demonstrated with the FOLFOX regimen (Bokemeyer et al, 2009; Douillard et al, 2010; Qin et al, 2016). In the NORDIC-VII study, a bolus 5-FU/FA regimen was used, and in the COIN trial, the majority of patients received capecitabine. Thus, there might be a positive pharmacodynamic synergism between the antiEGFR antibodies and 5-FU administered via the FOLFOX regimens (Harstrick et al, 1998; Skvortsov et al, 2008; Kim et al, 2009 Bijnsdorp et al, 2010), which was not achieved when the Nordic FLOX bolus regimen was used as the chemotherapy backbone.

In conclusion, the patient population of NORDIC-VII seems to be comparable with other cohorts of mCRC patients who start first-line therapy. The lack of effect of cetuximab when added to the Nordic FLOX regimen strengthens the notion that this combination is not favourable and strongly suggests a negative interaction between the FLOX regimen and cetuximab.

\section{ACKNOWLEDGEMENTS}

The NORDIC-VII study was supported by Merck KGaA, Darmstadt; Germany and Sanofi; Oslo, Norway. This work was supported by The Norwegian Cancer Society and The Swedish Cancer Society. Merck KGaA reviewed the manuscript for medical accuracy only before journal submission. We are fully responsible for the content of this manuscript, and the views and opinions described in the publication reflect solely those of the authors.

\section{CONFLICT OF INTEREST}

HS declared grants and personal fees from Novartis, Ipsen, Amgen, Merck, and Nordic Drugs, and personal fees from Celgene, Bayer, Roche, and Pfizer. PÖ declared grants and personal fees from Roche, Merck, Sanofi Oncology, Amgen, Bayer, Eli Lilly, and Nordic Drugs, and personal fees from Baxalta/Shire. The remaining authors declare no conflict of interest.

\section{REFERENCES}

Amado RG, Wolf M, Peeters M, Van Cutsem E, Siena S, Freeman DJ, Juan T, Sikorski R, Suggs S, Radinsky R, Patterson SD, Chang DD (2008) Wildtype KRAS is required for panitumumab efficacy in patients with metastatic colorectal cancer. J Clin Oncol 26: 1626-1634.

Bijnsdorp, Kruyt FA, Fukushima M, Smid K, Gokoel S, Peters GJ (2010) Molecular mechanism underlying the synergistic interaction between trifluorothymidine and the epidermal growth factor receptor inhibitor erlotinib in human colorectal cancer cell lines. Cancer Sci 101: 440-447.

Bokemeyer C, Bondarenko I, Makhson A, Hartmann JT, Aparicio J, de Braud F, Donea S, Ludwig H, Schuch G, Stroh C, Loos AH, Zubel A, Koralewski P (2009) Fluorouracil, leucovorin, and oxaliplatin with and without cetuximab in the first-line treatment of metastatic colorectal cancer. J Clin Oncol 27: 663-671.

Bokemeyer C, Kohne CH, Ciardiello F, Lenz HJ, Heinemann V, Klinkhardt U, Beier F, Duecker K, van Krieken JH, Tejpar S (2015) FOLFOX4 plus cetuximab treatment and RAS mutations in colorectal cancer. Eur J Cancer 51: $1243-1252$.

Cremolini C, Loupakis F, Antoniotti C, Lupi C, Sensi E, Lonardi S, Mezi S, Tomasello G, Ronzoni M, Zaniboni A, Tonini G, Carlomagno C, Allegrini G, Chiara S, D’Amico M, Granetto C, Cazzaniga M, Boni L, Fontanini G, Falcone A (2015) FOLFOXIRI plus bevacizumab versus FOLFIRI plus bevacizumab as first-line treatment of patients with metastatic colorectal cancer: updated overall survival and molecular subgroup analyses of the open-label, phase 3 TRIBE study. Lancet Oncol 16: 1306-1315.

Dahan L, Sadok A, Formento JL, Seitz JF, Kovacic H (2009) Modulation of cellular redox state underlies antagonism between oxaliplatin and cetuximab in human colorectal cancer cell lines. Br J Pharmacol 158: 610-620.

Douillard JY, Oliner KS, Siena S, Tabernero J, Burkes R, Barugel M, Humblet Y, Bodoky G, Cunningham D, Jassem J, Rivera F, Kocakova I, Ruff P, Blasinska-Morawiec M, Smakal M, Canon JL, Rother M, Williams R, Rong A, Wiezorek J, Sidhu R, Patterson SD (2013) Panitumumab-FOLFOX4 treatment and RAS mutations in colorectal cancer. N Engl J Med 369: 1023-1034.

Douillard JY, Siena S, Cassidy J, Tabernero J, Burkes R, Barugel M, Humblet Y, Bodoky G, Cunningham D, Jassem J, Rivera F, Kocakova I, Ruff P, Blasinska-Morawiec M, Smakal M, Canon JL, Rother M, Oliner KS, Wolf M, Gansert J (2010) Randomized, phase III trial of panitumumab with infusional fluorouracil, leucovorin, and oxaliplatin (FOLFOX4) versus FOLFOX4 alone as first-line treatment in patients with previously untreated metastatic colorectal cancer: the PRIME study. J Clin Oncol 28: 4697-4705.

Grothey A, Lenz HJ (2012) Explaining the unexplainable: EGFR antibodies in colorectal cancer. J Clin Oncol 30: 1735-1737.

Harstrick A, Gonzales A, Schleucher N, Vanhoefer U, Lu K, Formento JL, Milano G, Wilke H, Seeber S, Rustum Y (1998) Comparison between short or long exposure to 5 -fluorouracil in human gastric and colon cancer cell lines: biochemical mechanism of resistance. Anticancer Drugs 9: 625-634.

Karapetis CS, Khambata-Ford S, Jonker DJ, O'Callaghan CJ, Tu D, Tebbutt NC, Simes RJ, Chalchal H, Shapiro JD, Robitaille S, Price TJ, Shepherd L, Au HJ, Langer C, Moore MJ, Zalcberg JR (2008) K-ras mutations and benefit from cetuximab in advanced colorectal cancer. N Engl J Med 359: 1757-1765.

Kim HP, Yoon YK, Kim JW, Han SW, Hur HS, Park J, Lee JH, Oh DY, Im SA, Bang YJ, Kim TY (2009) Lapatinib, a dual EGFR and HER2 tyrosine kinase inhibitor, downregulates thymidylate synthase by inhibiting the nuclear translocation of EGFR and HER2. PloS One 4: e5933. 
Mahipal A, Grothey A (2016) Role of biologics in first-line treatment of colorectal cancer. J Oncol Pract 12: 1219-1228.

Maughan TS, Adams RA, Smith CG, Meade AM, Seymour MT, Wilson RH, Idziaszczyk S, Harris R, Fisher D, Kenny SL, Kay E, Mitchell JK, Madi A, Jasani B, James MD, Bridgewater J, Kennedy MJ, Claes B, Lambrechts D, Kaplan R, Cheadle JP (2011) Addition of cetuximab to oxaliplatin-based first-line combination chemotherapy for treatment of advanced colorectal cancer: results of the randomised phase 3 MRC COIN trial. Lancet 377: 2103-2114.

Primrose J, Falk S, Finch-Jones M, Valle J, O’Reilly D, Siriwardena A, Hornbuckle J, Peterson M, Rees M, Iveson T, Hickish T, Butler R, Stanton L, Dixon E, Little L, Bowers M, Pugh S, Garden OJ, Cunningham D, Maughan T, Bridgewater J (2014) Systemic chemotherapy with or without cetuximab in patients with resectable colorectal liver metastasis: the New EPOC randomised controlled trial. Lancet Oncol 15: 601-611.

Qin S, Xu J, Wang L, Cheng Y, Liu T, Chen J, Eggleton SP, Liu J, Li J (2016) Impact of tumor epidermal growth factor receptor (EGFR) status on the outcomes of first-line FOLFOX- $4 \pm$ cetuximab in patients (pts) with RAS-wild-type (wt) metastatic colorectal cancer (mCRC) in the randomized phase 3 TAILOR trial. Ann Oncol 27: iil41.

Rajagopalan H, Bardelli A, Lengauer C, Kinzler KW, Vogelstein B, Velculescu VE (2002) Tumorigenesis: RAF/RAS oncogenes and mismatch-repair status. Nature 418(6901): 934.

Santoro V, Jia R, Thompson H, Nijhuis A, Jeffery R, Kiakos K, Silver AR, Hartley JA, Hochhauser D (2015) Role of reactive oxygen species in the abrogation of oxaliplatin activity by cetuximab in colorectal cancer. J Natl Cancer Inst 108: djv394.

Skvortsov S, Sarg B, Lindner H, Lukas P, Hilbe W, Zwierzina H, Skvortsova I (2008) Cetuximab inhibits thymidylate synthase in colorectal cells expressing epidermal growth factor receptor. Proteomics Clin Appl 2: 908-914.

Sorbye H, Glimelius B, Berglund A, Fokstuen T, Tveit KM, Braendengen M, Ogreid D, Dahl O (2004) Multicenter phase II study of Nordic fluorouracil and folinic acid bolus schedule combined with oxaliplatin as first-line treatment of metastatic colorectal cancer. J Clin Oncol 22: 31-38.
Tejpar S, Stintzing S, Ciardiello F, Tabernero J, Van Cutsem E, Beier F, Esser R, Lenz HJ, Heinemann V (2017) Prognostic and predictive relevance of primary tumor location in patients with RAS wild-type metastatic colorectal cancer: retrospective analyses of the CRYSTAL and FIRE-3 trials. JAMA Oncol; 3: 194-201.

Tveit KM, Guren T, Glimelius B, Pfeiffer P, Sorbye H, Pyrhonen S, Sigurdsson F, Kure E, Ikdahl T, Skovlund E, Fokstuen T, Hansen F, Hofsli E, Birkemeyer E, Johnsson A, Starkhammar H, Yilmaz MK, Keldsen N, Erdal AB, Dajani O, Dahl O, Christoffersen T (2012) Phase III trial of cetuximab with continuous or intermittent fluorouracil, leucovorin, and oxaliplatin (Nordic FLOX) versus FLOX alone in first-line treatment of metastatic colorectal cancer: the NORDIC-VII study. J Clin Oncol 30: 1755-1762.

Van Cutsem E, Kohne CH, Hitre E, Zaluski J, Chang Chien CR, Makhson A, D’Haens G, Pinter T, Lim R, Bodoky G, Roh JK, Folprecht G, Ruff P, Stroh C, Tejpar S, Schlichting M, Nippgen J, Rougier P (2009) Cetuximab and chemotherapy as initial treatment for metastatic colorectal cancer. $N$ Engl J Med 360: 1408-1417.

Van Cutsem E, Lenz HJ, Kohne CH, Heinemann V, Tejpar S, Melezinek I, Beier F, Stroh C, Rougier P, van Krieken JH, Ciardiello F (2015) Fluorouracil, leucovorin, and irinotecan plus cetuximab treatment and RAS mutations in colorectal cancer. J Clin Oncol 33: 692-700.

Wasan H, Meade AM, Adams R, Wilson R, Pugh C, Fisher D, Sydes B, Madi A, Sizer B, Lowdell C, Middleton G, Butler R, Kaplan R, Maughan T (2014) Intermittent chemotherapy plus either intermittent or continuous cetuximab for first-line treatment of patients with KRAS wild-type advanced colorectal cancer (COIN-B): a randomised phase 2 trial. Lancet Oncol 15: 631-639.

cc)(1) (2) This work is licensed under the Creative Commons BY ${ }_{\text {NG }}$ Aa Attribution-Non-Commercial-Share Alike 4.0 International License. To view a copy of this license, visit http:// creativecommons.org/licenses/by-nc-sa/4.0/

(C) The Author(s) named above 2017

Supplementary Information accompanies this paper on British Journal of Cancer website (http://www.nature.com/bjc) 